Теорія Ймовір. та Матем. Статист. Вип. 80, 2009
Theor. Probability and Math. Statist.

No. 80, 2010, Pages 153-172

S 0094-9000(2010)00802-7

Article electronically published on August 20, 2010

\title{
CONVERGENCE OF OPTION REWARDS FOR MARKOV TYPE PRICE PROCESSES MODULATED BY STOCHASTIC INDICES. II
}

UDC 519.21

\author{
D. S. SILVESTROV, H. JÖNSSON, AND F. STENBERG
}

\begin{abstract}
A general price process represented by a two-component Markov process is considered. Its first component is interpreted as a price process and the second one as a stochastic index modulating the price component. American type options with pay-off functions, which admit upper bounds of a power type, are studied. Both the transition characteristics of the price processes and the pay-off functions are assumed to depend on a perturbation parameter $\delta \geq 0$ and to converge to the corresponding limit characteristics as $\delta \rightarrow 0$. In the first part of the paper, asymptotically uniform skeleton approximations connecting reward functionals for continuous and discrete time models are given. In the second part of the paper, these skeleton approximations are used for getting results about the convergence of reward functionals for American type options for perturbed price processes with discrete and continuous time. Examples related to modulated exponential price processes with independent increments are given.
\end{abstract}

The paper is a continuation of [1, where one can find an introduction to the topic, a survey of preceding works, a bibliography of related publications, a motivation for our studies, and comments concerning presented results. We continue our study of the optimal exercise of American type options under Markov price processes modulated by stochastic indices. The aim of the present paper is to obtain results on the convergence of option rewards for both discrete and continuous time models and to show how our general convergence results can be applied to exponential price processes with independent increments, exponential Lévy price processes modulated by semi-Markov stochastic indices, and to some other models.

\section{Convergence of ReWARds For Discrete time options}

In this section we give some conditions for the convergence of discrete time reward functionals $\Phi\left(\mathcal{M}_{\Pi, T}^{(\delta)}\right)$ for a given partition $\Pi=\left\{0=t_{0}<t_{1} \cdots<t_{N}=T\right\}$ of the interval $[0, T]$.

In this case, it is natural to use conditions expressed in terms of the probabilities of transitions between sequential moments of the partition $\Pi$ and in terms of the values of the pay-off functions at the moments belonging to this partition.

In the case of continuous time, the derivatives of the pay-off functions are involved in condition $\mathbf{A}_{\mathbf{1}}$ (see [1]). The corresponding assumptions imply the continuity of the pay-off functions. These assumptions play an essential role in the proof of Theorem 1 concerning skeleton approximations.

2000 Mathematics Subject Classification. Primary 60J05, 60H10; Secondary 91B28, 91B70.

Key words and phrases. Reward, convergence, optimal stopping, American option, skeleton approximation, Markov process, price process, modulation, stochastic index. 
In the case of discrete time, the derivatives of the pay-off functions are not involved in the assumptions. In this case, the pay-off functions can be discontinuous.

We replace $\mathbf{A}_{\mathbf{1}}$ by a simpler condition:

$\mathbf{A}_{\mathbf{2}}$ : There exists $\delta_{0}>0$ such that

$$
g^{(\delta)}\left(t_{n}, s\right) \leq K_{6}+K_{7} s^{\gamma}
$$

for all $0 \leq \delta \leq \delta_{0}, n=0, \ldots, N$, and $s \in(0, \infty)$, where $\gamma \geq 1$ and $K_{6}, K_{7}<\infty$ are some constants.

We also need an assumption for the convergence of the pay-off functions. We require the locally uniform convergence for the pay-off functions on some sets. It will be assumed later that the corresponding limit transition probabilities of these sets are equal to 1 and that the limit of the initial distribution exists:

$\mathbf{A}_{3}$ : For every $n=0, \ldots, N$, there exists a measurable set $\mathbb{S}_{t_{n}} \subseteq(0, \infty)$ such that $g^{(\delta)}\left(t_{n}, s_{\delta}\right) \rightarrow g^{(0)}\left(t_{n}, s\right)$ as $\delta \rightarrow 0$ for an arbitrary $s_{\delta} \rightarrow s \in \mathbb{S}_{t_{n}}$.

Put $\mathbb{V}_{t_{n}}=\mathbb{S}_{t_{n}} \times \mathbb{X}$.

Condition $\mathbf{A}_{\mathbf{3}}$ can be rewritten as follows in terms of the function $g^{(\delta)}\left(t, e^{y}\right)$, where $(t, y) \in[0, \infty) \times \mathbb{R}_{1}:$

$\mathbf{A}_{3}^{\prime}$ : For every $n=0, \ldots, N$, there exists a measurable set $\mathbb{Y}_{t_{n}}^{\prime} \subseteq \mathbb{R}_{1}$ such that $g^{(\delta)}\left(t_{n}, e^{y_{\delta}}\right) \rightarrow g^{(0)}\left(t_{n}, e^{y}\right)$ as $\delta \rightarrow 0$ for an arbitrary $y_{\delta} \rightarrow y \in \mathbb{Y}_{t_{n}}^{\prime}$.

It is obvious that the sets $\mathbb{S}_{t_{n}}$ and $\mathbb{Y}_{t_{n}}^{\prime}$ are such that $\mathbb{Y}_{t_{n}}^{\prime}=\ln \mathbb{S}_{t_{n}}=\left\{y=\ln s, s \in \mathbb{S}_{t_{n}}\right\}$ for all $n=0, \ldots, N$.

Typical examples of the sets $\overline{\mathbb{Y}}_{t_{n}}^{\prime}$ are presented by empty, finite, or countable sets. For example, let the pay-off functions $g^{(\delta)}\left(t, e^{y}\right)$ be monotone in $y$ and let $\mathbb{Y}_{t_{n}}^{*}$ be some countable dense sets in $\mathbb{R}_{1}$. Then the point-wise convergence $g^{(\delta)}\left(t, e^{y}\right) \rightarrow g^{(0)}\left(t, e^{y}\right)$ as $\delta \rightarrow 0, y \in \mathbb{Y}_{t_{n}}^{*}$, and for all $n=0, \ldots, N$ implies the locally uniform convergence required in condition $\mathbf{A}_{\mathbf{3}}^{\prime}$ where $\mathbb{Y}_{t_{n}}^{\prime}, n=0, \ldots, N$, are the sets of points of continuity for $g^{(0)}\left(t_{n}, e^{y}\right)$ considered as functions of the variable $y$. Since these functions are monotone, $\overline{\mathbb{Y}}_{t_{n}}^{\prime}$ are at most countable sets.

The symbol $\Rightarrow$ is used below to denote the weak convergence of probability measures or the weak convergence of the corresponding random variables.

We need some conditions imposed on the convergence of the transition probabilities of price processes between sequential moments of the partition

$$
\Pi=\left\{0=t_{0}<t_{1} \cdots<t_{N}=T\right\},
$$

namely

$\mathbf{B}_{1}$ : There exist measurable sets $\mathbb{Z}_{t_{n}} \subseteq \mathbb{Z}, n=0, \ldots, N$, such that

(a) $P^{(\delta)}\left(t_{n}, z_{\delta}, t_{n+1}, \cdot\right) \Rightarrow P^{(0)}\left(t_{n}, z, t_{n+1}, \cdot\right)$ as $\delta \rightarrow 0$ for an arbitrary

$$
z_{\delta} \rightarrow z \in \mathbb{Z}_{t_{n}} \quad \text { as } \delta \rightarrow 0
$$

and all $n=0, \ldots, N-1$;

(b) $P^{(0)}\left(t_{n}, z, t_{n+1}, \mathbb{Z}_{t_{n+1}}^{\prime} \cap \mathbb{Z}_{t_{n+1}}\right)=1$ for all $z \in \mathbb{Z}_{t_{n}}$ and $n=0, \ldots, N-1$, where $\mathbb{Z}_{t_{n}}^{\prime}=\mathbb{Y}_{t_{n}}^{\prime} \times \mathbb{X}$ and $\mathbb{Y}_{t_{n+1}}^{\prime}$ are the sets introduced in condition $\mathbf{A}_{\mathbf{3}}^{\prime}$.

A typical example is given by the sets $\overline{\mathbb{Z}}_{t_{n}}^{\prime} \cup \overline{\mathbb{Z}}_{t_{n}}=\varnothing$. In this case, condition $\mathbf{B}_{\mathbf{1}}$ (b) automatically holds. Another typical example arises if $\overline{\mathbb{Y}}_{t_{n}}^{\prime}$ and $\overline{\mathbb{Y}}_{t_{n}}$ are at most countable sets and $\mathbb{Z}_{t_{n}}^{\prime}=\mathbb{Y}_{t_{n}}^{\prime} \times \mathbb{X}$ and $\mathbb{Z}_{t_{n}}=\mathbb{Y}_{t_{n}} \times \mathbb{X}$. In this case, the assumption that the measures $P^{(0)}(t, z, t+u, A \times \mathbb{X}), A \in \mathcal{B}_{1}$, have no atoms implies condition $\mathbf{B}_{\mathbf{1}}(\mathbf{b})$. 
As far as the condition of the convergence of the initial distributions is concerned, we shall require the weak convergence of the initial distributions to some distribution concentrated at the intersections of the sets of convergence for the corresponding transition probabilities and pay-off functions, namely

$\mathbf{B}_{\mathbf{2}}$ : (a) $P^{(\delta)}(\cdot) \Rightarrow P^{(0)}(\cdot)$ as $\delta \rightarrow 0$;

(b) $P^{(0)}\left(\mathbb{Z}_{t_{0}}^{\prime} \cap \mathbb{Z}_{t_{0}}\right)=1$, where $\mathbb{Z}_{t_{0}}^{\prime}$ and $\mathbb{Z}_{t_{0}}$ are the sets introduced in conditions $\mathbf{A}_{\mathbf{2}}^{\prime}$ and $\mathbf{B}_{\mathbf{1}}$.

A typical example is given by $\overline{\mathbb{Z}}_{t_{0}}^{\prime} \cup \overline{\mathbb{Z}}_{t_{0}}=\varnothing$. In this case, condition $\mathbf{B}_{\mathbf{2}}$ (b) automatically holds. Another typical example is $\mathbb{Z}_{t_{0}}^{\prime}=\mathbb{Y}_{t_{0}}^{\prime} \times \mathbb{X}$ and $\mathbb{Z}_{t_{0}}=\mathbb{Y}_{t_{0}} \times \mathbb{X}$, where $\overline{\mathbb{Y}}_{t_{0}}^{\prime}$ and $\overline{\mathbb{Y}}_{t_{0}}$ are at most finite or countable sets. In this case, the assumption that the measure $P^{(0)}(A \times \mathbb{X}), A \in \mathcal{B}_{1}$, has no atoms implies that condition $\mathbf{B}_{\mathbf{2}}$ (b) holds.

Condition $\mathbf{B}_{\mathbf{2}}$ holds, for example, if the initial distributions $P^{(\delta)}(A)=\chi_{A}\left(z_{0}\right), \delta \geq 0$, are concentrated at a point $z_{0} \in \mathbb{Z}_{t_{0}}^{\prime} \cap \mathbb{Z}_{t_{0}}$. This condition also holds if the initial distributions are such that $P^{(\delta)}(A)=\chi_{A}\left(z_{\delta}\right)$ for $\delta \geq 0$, where $z_{\delta} \rightarrow z_{0}$ as $\delta \rightarrow 0$ and $z_{0} \in \mathbb{Z}_{t_{0}}^{\prime} \cap \mathbb{Z}_{t_{0}}$

We also replace $\mathbf{C}_{\mathbf{1}}$ by a simpler condition:

$\mathbf{C}_{\mathbf{3}}: \varlimsup_{\delta \rightarrow 0} \sup _{z \in \mathbb{Z}} \mathrm{E}_{z, t_{n}}\left(e^{\beta\left|Y^{(\delta)}\left(t_{n+1}\right)-Y^{(\delta)}\left(t_{n}\right)\right|}-1\right)<\infty, n=0, \ldots, N-1$, for some $\beta>\gamma$, where $\gamma$ is the parameter introduced in condition $\mathbf{A}_{\mathbf{2}}$.

Condition $\mathbf{C}_{\mathbf{2}}$ does not change and becomes of the following form:

$\mathbf{C}_{\mathbf{4}}: \varlimsup_{\lim _{\delta \rightarrow 0}} \mathrm{E} e^{\beta\left|Y^{(\delta)}\left(t_{0}\right)\right|}<\infty$, where $\beta$ is the parameter introduced in condition $\mathbf{C}_{\mathbf{3}}$.

The following theorem is the second main result of the present paper.

Theorem 2. Let conditions $\mathbf{A}_{\mathbf{2}}, \mathbf{A}_{\mathbf{3}}, \mathbf{B}_{\mathbf{1}}, \mathbf{B}_{\mathbf{2}}, \mathbf{C}_{\mathbf{3}}$, and $\mathbf{C}_{\mathbf{4}}$ hold. Then, the following asymptotic relation holds for the partition $\Pi=\left\{0=t_{0}<t_{1} \cdots<t_{N}=T\right\}$ of the interval $[0, T]$ :

$$
\Phi\left(\mathcal{M}_{\Pi, T}^{(\delta)}\right) \rightarrow \Phi\left(\mathcal{M}_{\Pi, T}^{(0)}\right) \quad \text { as } \delta \rightarrow 0 .
$$

Proof. We improve the method based on the recursive asymptotic analysis of reward functions used in Jönsson [4].

The reward functions are defined by the following recursive relations,

$$
w^{(\delta)}\left(t_{N}, z\right)=g^{(\delta)}\left(t_{N}, e^{y}\right), \quad z=(y, x) \in \mathbb{Z},
$$

and, for $n=0, \ldots, N-1$,

$$
w^{(\delta)}\left(t_{n}, z\right)=\max \left(g^{(\delta)}\left(t_{n}, e^{y}\right), \mathrm{E}_{z, t_{n}} w^{(\delta)}\left(t_{n+1}, Z^{(\delta)}\left(t_{n+1}\right)\right)\right), \quad z=(y, x) \in \mathbb{Z} .
$$

It follows from general results of Chow, Robbins, and Siegmund [2] and of Shiryaev [10] on optimal stopping problems for discrete time Markov processes that the reward functional is such that

$$
\Phi\left(\mathcal{M}_{\Pi, T}^{(\delta)}\right)=\mathrm{E} w^{(\delta)}\left(t_{0}, Z^{(\delta)}(0)\right) .
$$

By definition, the reward functions are nonnegative, that is, $w^{(\delta)}\left(t_{n}, z\right) \geq 0$ for $z \in \mathbb{Z}$ and $n=0, \ldots, N$.

Condition $\mathbf{C}_{3}$ implies that there exist constants $L_{10}<\infty$ and $\delta_{2} \leq \delta_{0}$ such that for all $n=0, \ldots, N-1$ and $\delta \leq \delta_{2}$,

$$
\sup _{z \in \mathbb{Z}} \mathrm{E}_{z, t_{n}}\left(e^{\beta\left|Y^{(\delta)}\left(t_{n+1}\right)-Y^{(\delta)}\left(t_{n}\right)\right|}-1\right) \leq L_{10} .
$$

Condition $\mathbf{C}_{4}$ implies that $\delta_{2}$ can be chosen in such a way that, for some constant $L_{11}<\infty$, the following inequality holds for $\delta \leq \delta_{2}$ :

$$
\mathrm{E} e^{\beta\left|Y^{(\delta)}(0)\right|} \leq L_{11} \text {. }
$$


Condition $\mathbf{A}_{2}$ directly implies that the following upper bound for the reward function $w^{(\delta)}\left(t_{N}, z\right)$ holds for $\delta \leq \delta_{2}$ :

$$
w^{(\delta)}\left(t_{N}, z\right) \leq L_{1, N}+L_{2, N} e^{\gamma|y|}, \quad z=(y, x) \in \mathbb{Z},
$$

where

$$
L_{1, N}=K_{6}, \quad L_{2, N}=K_{7}<\infty .
$$

According to condition $\mathbf{A}_{\mathbf{3}}^{\prime}$,

$$
w^{(\delta)}\left(t_{N}, z_{\delta}\right) \rightarrow w^{(0)}\left(t_{N}, z_{0}\right) \quad \text { as } \delta \rightarrow 0
$$

for an arbitrary $z_{\delta} \rightarrow z_{0} \in \mathbb{Z}_{t_{N}}^{\prime} \cap \mathbb{Z}_{t_{N}}$ as $\delta \rightarrow 0$.

Now we prove relations similar to (5), (6), and (7) for the reward functions

$$
w^{(\delta)}\left(t_{N-1}, z\right) \text {. }
$$

Using relation (5) for $z=(y, x) \in \mathbb{Z}$ and $\delta \leq \delta_{2}$, we get

$$
\begin{aligned}
\mathrm{E}_{z, t_{N-1}} g^{(\delta)}\left(t_{N}, e^{Y^{(\delta)}\left(t_{N}\right)}\right) & \leq L_{1, N}+L_{2, N} \mathrm{E}_{z, t_{N-1}} e^{\gamma\left|Y^{(\delta)}\left(t_{N}\right)\right|} \\
& \leq L_{1, N}+L_{2, N} \mathrm{E}_{z, t_{N-1}} e^{\gamma|y|} e^{\gamma\left|Y^{(\delta)}\left(t_{N}\right)-y\right|} \\
& \leq L_{1, N}+L_{2, N} e^{\gamma|y|} \mathrm{E}_{z, t_{N-1}} e^{\gamma\left|Y^{(\delta)}\left(t_{N}\right)-Y^{(\delta)}\left(t_{N-1}\right)\right|} \\
& \leq L_{1, N}+L_{2, N}\left(L_{10}+1\right) e^{\gamma|y|} .
\end{aligned}
$$

Relation (8) implies that

$$
\begin{aligned}
w^{(\delta)}\left(t_{N-1}, z\right) & =\max \left(g^{(\delta)}\left(t_{N-1}, e^{y}\right), \mathrm{E}_{z, t} w^{(\delta)}\left(t_{N}, Z^{(\delta)}\left(t_{N}\right)\right)\right) \\
& \leq K_{6}+K_{7} e^{\gamma|y|}+L_{1, N}+L_{2, N}\left(L_{10}+1\right) e^{\gamma|y|} \\
& \leq L_{1, N-1}+L_{2, N-1} e^{\gamma|y|}
\end{aligned}
$$

for $z=(y, x) \in \mathbb{Z}$ and $\delta \leq \delta_{2}$, where

$$
L_{1, N-1}=K_{6}+L_{1, N}, \quad L_{2, N-1}=K_{7}+L_{2, N}\left(L_{10}+1\right)<\infty .
$$

For every $n=0, \ldots, N-1$ and $z \in \mathbb{Z}$, we introduce the random variables

$$
Z_{n}^{(\delta)}(z)=\left(Y_{n}^{(\delta)}(z), X_{n}^{(\delta)}(z)\right)
$$

such that $\mathrm{P}\left\{Z_{n}^{(\delta)}(z) \in A\right\}=P^{(\delta)}\left(t_{n}, z, t_{n+1}, A\right), A \in \mathcal{B}_{\mathbb{Z}}$.

Now we check that

$$
w^{(\delta)}\left(t_{N}, Z_{N-1}^{(\delta)}\left(z_{\delta}\right)\right) \Rightarrow w^{(0)}\left(t_{N}, Z_{N-1}^{(0)}\left(z_{0}\right)\right) \quad \text { as } \delta \rightarrow 0
$$

for an arbitrary $z_{\delta} \rightarrow z_{0} \in \mathbb{Z}_{t_{N-1}}^{\prime} \cap \mathbb{Z}_{t_{N-1}}$ as $\delta \rightarrow 0$.

Relation (11) follows from general results on the weak convergence for compositions of random functions obtained in Silvestrov [1]. Note that the functions $w^{(\delta)}\left(t_{N}, \cdot\right)$ in the composition on the right hand side of (11) are non-random. This allows us to provide a simpler proof of this relation.

Consider an arbitrary sequence $\delta_{k} \rightarrow \delta_{0}=0$ as $k \rightarrow \infty$. According to condition $\mathbf{B}_{\mathbf{1}}$,

(a) the random variables $Z_{N-1}^{\left(\delta_{k}\right)}\left(z_{\delta_{k}}\right)$ weakly converge to $Z_{N-1}^{\left(\delta_{0}\right)}\left(z_{\delta_{0}}\right)$ as $k \rightarrow \infty$ for an arbitrary $z_{\delta_{k}} \rightarrow z_{\delta_{0}} \in \mathbb{Z}_{t_{N-1}}^{\prime} \cap \mathbb{Z}_{t_{N-1}}$ as $k \rightarrow \infty$,

and

(b) $\mathrm{P}\left\{Z_{N-1}^{\left(\delta_{0}\right)}\left(z_{\delta_{0}}\right) \in \mathbb{Z}_{t_{N}}^{\prime} \cap \mathbb{Z}_{t_{N}}\right\}=1$.

By the Skorokhod [14] representation theorem, 
(c) one can construct random variables $\tilde{Z}_{N-1}^{\left(\delta_{k}\right)}\left(z_{\delta_{k}}\right), k=0,1, \ldots$, on a common probability space $(\Omega, \mathcal{F}, P)$ such that

$$
\mathrm{P}\left\{\tilde{Z}_{N-1}^{\left(\delta_{k}\right)}\left(z_{\delta_{k}}\right) \in A\right\}=\mathrm{P}\left\{Z_{N-1}^{\left(\delta_{k}\right)}\left(z_{\delta_{k}}\right) \in A\right\}
$$

for $A \in \mathcal{B}_{\mathbb{Z}}$ and for all $k=0,1, \ldots$, and

(d) $\tilde{Z}_{N-1}^{\left(\delta_{k}\right)}\left(z_{\delta_{k}}\right) \stackrel{\text { a.s. }}{\longrightarrow} \tilde{Z}_{N-1}^{\left(\delta_{0}\right)}\left(z_{\delta_{0}}\right)$ as $k \rightarrow \infty$.

Let

$$
A_{N-1}=\left\{\omega \in \Omega: \tilde{Z}_{N-1}^{\left(\delta_{k}\right)}\left(z_{\delta_{k}}, \omega\right) \rightarrow \tilde{Z}_{N-1}^{\left(\delta_{0}\right)}\left(z_{\delta_{0}}, \omega\right) \text { as } k \rightarrow \infty\right\}
$$

and

$$
B_{N-1}=\left\{\omega \in \Omega: \tilde{Z}_{N-1}^{\left(\delta_{0}\right)}\left(z_{\delta_{0}}, \omega\right) \in \mathbb{Z}_{t_{N}}^{\prime} \cap \mathbb{Z}_{t_{N}}\right\} .
$$

Relation (d) implies that $\mathrm{P}\left(A_{N-1}\right)=1$. Relations (b) and (c) imply that $\mathrm{P}\left(B_{N-1}\right)=1$. The latter two equalities yield $\mathrm{P}\left(A_{N-1} \cap B_{N-1}\right)=1$. By relation (7) and the definition of the sets $A_{N-1}$ and $B_{N-1}$, we get the convergence

$$
w^{\left(\delta_{k}\right)}\left(t_{N}, \tilde{Z}_{N-1}^{\left(\delta_{k}\right)}\left(z_{\delta_{k}}, \omega\right)\right) \rightarrow w^{\left(\delta_{0}\right)}\left(t_{N}, \tilde{Z}_{N-1}^{\left(\delta_{0}\right)}\left(z_{\delta_{0}}, \omega\right)\right) \quad \text { as } k \rightarrow \infty
$$

for $\omega \in A_{N-1} \cap B_{N-1}$. Thus,

(e) the random variables converge almost surely, that is,

$$
w^{\left(\delta_{k}\right)}\left(t_{N}, \tilde{Z}_{N-1}^{\left(\delta_{k}\right)}\left(z_{\delta_{k}}\right)\right) \stackrel{\text { a.s. }}{\longrightarrow} w^{\left(\delta_{0}\right)}\left(t_{N}, \tilde{Z}_{N-1}^{\left(\delta_{0}\right)}\left(z_{\delta_{0}}\right)\right) \quad \text { as } k \rightarrow \infty .
$$

Relation (c) implies that

(f) for $A \in \mathcal{B}_{\mathbb{Z}}$ and all $k=0,1, \ldots$,

$$
\mathrm{P}\left\{w^{\left(\delta_{k}\right)}\left(t_{N}, \tilde{Z}_{N-1}^{\left(\delta_{k}\right)}\left(z_{\delta_{k}}\right)\right) \in A\right\}=\mathrm{P}\left\{w^{\left(\delta_{k}\right)}\left(t_{N}, Z_{N-1}^{\left(\delta_{k}\right)}\left(z_{\delta_{k}}\right)\right) \in A\right\} .
$$

Relations (e) and (f) imply that

(g) the random variables $w^{\left(\delta_{k}\right)}\left(t_{N}, Z_{N-1}^{\left(\delta_{k}\right)}\left(z_{\delta_{k}}\right)\right)$ weakly converge to

$$
w^{\left(\delta_{0}\right)}\left(t_{N}, Z_{N-1}^{\left(\delta_{0}\right)}\left(z_{\delta_{0}}\right)\right) \quad \text { as } k \rightarrow \infty .
$$

Since the sequence $\delta_{k} \rightarrow \delta_{0}$ is arbitrary, relation (g) proves convergence (11). Using inequality (9) and condition $\mathbf{C}_{\mathbf{3}}$ we get

$$
\begin{aligned}
\mathrm{E}\left(w^{(\delta)}\left(t_{N}, Z_{N-1}^{(\delta)}\left(z_{\delta}\right)\right)\right)^{\frac{\beta}{\gamma}}=\mathrm{E}_{z_{\delta}, t_{N-1}}\left(w^{(\delta)}\left(t_{N}, Z^{(\delta)}\left(t_{N}\right)\right)\right)^{\frac{\beta}{\gamma}} \\
\quad \leq \mathrm{E}_{z_{\delta}, t_{N-1}}\left(L_{1, N}+L_{2, N} e^{\gamma\left|Y^{(\delta)}\left(t_{N}\right)\right|}\right)^{\frac{\beta}{\gamma}} \\
\quad \leq 2^{\frac{\beta}{\gamma}-1}\left(\left[L_{1, N}\right]^{\frac{\beta}{\gamma}}+\left[L_{2, N}\right]^{\frac{\beta}{\gamma}} \mathrm{E}_{z_{\delta}, t_{N-1}} e^{\beta\left|y_{\delta}\right|} e^{\beta\left|Y^{(\delta)}\left(t_{N}\right)-y_{\delta}\right|}\right) \\
\quad \leq 2^{\frac{\beta}{\gamma}-1}\left(\left[L_{1, N}\right]^{\frac{\beta}{\gamma}}+\left[L_{2, N}\right]^{\frac{\beta}{\gamma}}\left(L_{10}+1\right) e^{\beta\left|y_{\delta}\right|}\right)
\end{aligned}
$$

for an arbitrary sequence

$$
z_{\delta}=\left(y_{\delta}, x_{\delta}\right) \rightarrow z_{0}=\left(y_{0}, x_{0}\right) \in \mathbb{Z}_{t_{N-1}}^{\prime} \cap \mathbb{Z}_{t_{N-1}} \quad \text { as } \delta \rightarrow 0,
$$

and for $\delta \leq \delta_{2}$. Therefore,

$$
\varlimsup_{\delta \rightarrow 0} \mathrm{E}\left(w^{(\delta)}\left(t_{N}, Z_{N-1}^{(\delta)}\left(z_{\delta}\right)\right)\right)^{\frac{\beta}{\gamma}}<\infty .
$$


Relations (11) and (13) imply that

$$
\mathrm{E}_{z_{\delta}, t_{N-1}} w^{(\delta)}\left(t_{N}, Z^{(\delta)}\left(t_{N}\right)\right) \rightarrow \mathrm{E}_{z_{0}, t_{N-1}} w^{(0)}\left(t_{N}, Z^{(0)}\left(t_{N}\right)\right) \quad \text { as } \delta \rightarrow 0
$$

for an arbitrary sequence $z_{\delta} \rightarrow z_{0} \in \mathbb{Z}_{t_{N-1}}^{\prime} \cap \mathbb{Z}_{t_{N-1}}$ as $\delta \rightarrow 0$.

Relation (14) and condition $\mathbf{A}_{\mathbf{3}}^{\prime}$ yield

$$
\begin{gathered}
w^{(\delta)}\left(t_{N-1}, z_{\delta}\right)=g^{(\delta)}\left(t_{N-1}, e^{y_{\delta}}\right) \vee \mathrm{E}_{z_{\delta}, t_{N-1}} w^{(\delta)}\left(t_{N}, Z^{(\delta)}\left(t_{N}\right)\right) \\
\rightarrow w^{(0)}\left(t_{N-1}, z_{0}\right)=g^{(0)}\left(t_{N-1}, e^{y_{0}}\right) \vee \mathrm{E}_{z_{0}, t_{N-1}} w^{(0)}\left(t_{N}, Z^{(0)}\left(t_{N}\right)\right) \\
\text { as } \delta \rightarrow 0
\end{gathered}
$$

for an arbitrary sequence

$$
z_{\delta}=\left(y_{\delta}, x_{\delta}\right) \rightarrow z_{0}=\left(y_{0}, x_{0}\right) \in \mathbb{Z}_{t_{N-1}}^{\prime} \cap \mathbb{Z}_{t_{N-1}} \quad \text { as } \delta \rightarrow 0 .
$$

Relations (9), (10), and (15) are analogues of relations (5), (6), and (7), respectively. By repeating the recursive procedure described above we finally get

$$
w^{(\delta)}\left(t_{n}, z\right) \leq L_{1, n}+L_{2, n} e^{\gamma|y|}, \quad z=(y, x) \in \mathbb{Z},
$$

for all $n=0,1, \ldots, N$, all $\delta \leq \delta_{2}$, and for some constants,

$$
L_{1, n}, L_{2, n}<\infty \text {. }
$$

Moreover

$$
w^{(\delta)}\left(t_{n}, z_{\delta, n}\right) \rightarrow w^{(0)}\left(t_{n}, z_{0, n}\right) \quad \text { as } \delta \rightarrow 0
$$

for an arbitrary sequence $z_{\delta, n} \rightarrow z_{0, n}$ as $\delta \rightarrow 0$, where $z_{0, n} \in \mathbb{Z}_{t_{n}}^{\prime} \cap \mathbb{Z}_{t_{n}}$, and for all $n=0,1, \ldots, N$.

Consider an arbitrary sequence $\delta_{k} \rightarrow \delta_{0}=0$ as $k \rightarrow \infty$. According to condition $\mathbf{B}_{\mathbf{2}}$,

(h) the random variables are such that $Z^{\left(\delta_{k}\right)}(0) \Rightarrow Z^{\left(\delta_{0}\right)}(0)$ as $k \rightarrow \infty$,

(i) and

$$
\mathrm{P}\left\{Z^{\left(\delta_{0}\right)}(0) \in \mathbb{Z}_{t_{0}}^{\prime} \cap \mathbb{Z}_{t_{0}}\right\}=1 .
$$

According to Skorokhod's representation theorem,

(j) one can construct random variables $\tilde{Z}^{\left(\delta_{k}\right)}(0), k=0,1, \ldots$, on a common probability space $(\Omega, \mathcal{F}, \mathrm{P})$ such that

$$
\mathrm{P}\left\{\tilde{Z}^{\left(\delta_{k}\right)}(0) \in A\right\}=\mathrm{P}\left\{Z^{\left(\delta_{k}\right)}(0) \in A\right\}, \quad A \in \mathcal{B}_{\mathbb{Z}},
$$

for all $k=0,1, \ldots$,

and

(k) $\tilde{Z}^{\left(\delta_{k}\right)}(0) \stackrel{\text { a.s. }}{\longrightarrow} \tilde{Z}^{\left(\delta_{0}\right)}(0)$ as $k \rightarrow \infty$.

Put

$$
A=\left\{\omega \in \Omega: \tilde{Z}^{\left(\delta_{k}\right)}(0, \omega) \rightarrow \tilde{Z}^{\left(\delta_{0}\right)}(0, \omega) \text { as } k \rightarrow \infty\right\}
$$

and $B=\left\{\omega \in \Omega: \tilde{Z}^{\left(\delta_{0}\right)}(0, \omega) \in \mathbb{Z}_{t_{0}}^{\prime} \cap \mathbb{Z}_{t_{0}}\right\}$. Relation $(\mathrm{k})$ implies that $\mathrm{P}(A)=1$. Relations (i) and (j) prove that $\mathrm{P}(B)=1$. The latter two equalities yield

$$
\mathrm{P}(A \cap B)=1 \text {. }
$$

By condition $\mathbf{B}_{\mathbf{2}}$, relation (18), and the definition of sets $A$ and $B$, we prove the convergence

$$
w^{\left(\delta_{k}\right)}\left(t_{0}, \tilde{Z}^{\left(\delta_{k}\right)}(0, \omega)\right) \rightarrow w^{\left(\delta_{0}\right)}\left(t_{0}, \tilde{Z}^{\left(\delta_{0}\right)}(0, \omega)\right) \quad \text { as } k \rightarrow \infty
$$

for $\omega \in A \cap B$. Thus, 
(l) the random variables $w^{\left(\delta_{k}\right)}\left(t_{0}, \tilde{Z}^{\left(\delta_{k}\right)}(0)\right)$ converge almost surely to

$$
w^{\left(\delta_{0}\right)}\left(t_{0}, \tilde{Z}^{\left(\delta_{0}\right)}(0)\right) \quad \text { as } k \rightarrow \infty \text {. }
$$

Relation (j) implies that

(m) for $A \in \mathcal{B}_{\mathbb{Z}}$ and all $k=0,1, \ldots$,

$$
\mathrm{P}\left\{w^{\left(\delta_{k}\right)}\left(t_{0}, \tilde{Z}^{\left(\delta_{k}\right)}(0)\right) \in A\right\}=\mathrm{P}\left\{w^{\left(\delta_{k}\right)}\left(t_{0}, Z^{\left(\delta_{k}\right)}(0)\right) \in A\right\} .
$$

Relations (l) and (m) give us

(n) the random variables $w^{\left(\delta_{k}\right)}\left(t_{N}, Z^{\left(\delta_{k}\right)}(0)\right)$ weakly converge to $w^{\left(\delta_{0}\right)}\left(t_{N}, Z^{\left(\delta_{0}\right)}(0)\right)$ as $k \rightarrow \infty$.

Since the sequence $\delta_{k} \rightarrow \delta_{0}$ is arbitrary, relation (n) implies that

$$
w^{(\delta)}\left(t_{0}, Z^{(\delta)}(0)\right) \Rightarrow w^{(0)}\left(t_{0}, Z^{(0)}(0)\right) \quad \text { as } \delta \rightarrow 0
$$

Using inequality (16) and condition $\mathbf{C}_{4}$, we get

$$
\begin{aligned}
\mathrm{E}\left(w^{(\delta)}\left(t_{0}, Z^{(\delta)}(0)\right)\right)^{\frac{\beta}{\gamma}} & \leq \mathrm{E}\left(L_{1,0}+L_{2,0} e^{\gamma\left|Y^{(\delta)}(0)\right|}\right)^{\frac{\beta}{\gamma}} \\
& \leq 2^{\frac{\beta}{\gamma}-1}\left(\left(L_{1,0}\right)^{\frac{\beta}{\gamma}}+\left(L_{2,0}\right)^{\frac{\beta}{\gamma}} \mathrm{E} e^{\beta\left|Y^{(\delta)}(0)\right|}\right) \\
& \leq 2^{\frac{\beta}{\gamma}-1}\left(\left(L_{1,0}\right)^{\frac{\beta}{\gamma}}+\left(L_{2,0}\right)^{\frac{\beta}{\gamma}} L_{11}\right)
\end{aligned}
$$

for $\delta \leq \delta_{3}$. Therefore,

$$
\varlimsup_{\delta \rightarrow 0} \mathrm{E}\left(w^{(\delta)}\left(t_{0}, Z^{(\delta)}(0)\right)\right)^{\frac{\beta}{\gamma}}<\infty .
$$

We deduce from relations (19) and (21) that

$$
\mathrm{E} w^{(\delta)}\left(t_{0}, Z^{(\delta)}(0)\right) \rightarrow \mathrm{E} w^{(0)}\left(t_{0}, Z^{(0)}(0)\right) \quad \text { as } \delta \rightarrow 0
$$

Now we obtain relation (1) from formula (2) and convergence (22).

The proof of Theorem 2 is complete.

In order to prove the convergence of the reward functionals $\Phi\left(\mathcal{M}_{\Pi_{N}, T}^{(\delta)}\right)$ for any partition $\Pi_{N}$ of the interval $[0, T]$, one can assume the conditions of Theorem 3 for any partition of this interval. Note that these conditions do not involve the derivatives of the pay-off functions. In this case, the pre-limit and the limit pay-off functions can be discontinuous.

\section{Convergence of ReWArds for COntinuous time Price Processes}

As mentioned above, the pay-off functions can be discontinuous in the case of discrete time. In the case of continuous time, the derivatives of pay-off functions are involved in condition $\mathbf{A}_{1}$ and thus the corresponding assumptions imply the continuity of the pay-off functions.

This allows us to reduce the assumption concerning the convergence of pay-off functions to the assumption of pointwise convergence:

$\mathbf{A}_{4}: g^{(\delta)}(t, s) \rightarrow g^{(0)}(t, s)$ as $\delta \rightarrow 0$ for all $(t, s) \in[0, T] \times(0, \infty)$.

Condition $\mathbf{A}_{\mathbf{4}}$ can be rewritten in terms of the function

$$
g^{(\delta)}\left(t, e^{y}\right), \quad(t, y) \in[0, \infty) \times \mathbb{R}_{1},
$$

as follows:

$\mathbf{A}_{\mathbf{4}}^{\prime}: g^{(\delta)}\left(t, e^{y}\right) \rightarrow g^{(0)}\left(t, e^{y}\right)$ as $\delta \rightarrow 0$ for all $(t, y) \in[0, T] \times \mathbb{R}_{1}$. 
Below we list conditions on the transition probabilities and initial distributions of the process $Z^{(\delta)}(t)$.

The first condition concerns the weak convergence of the transition probabilities. We assume that the transition probabilities are locally uniform with respect to the initial states belonging to some sets. The second part of the condition below means that the corresponding limit measures are concentrated on these sets:

$\mathbf{B}_{3}$ : There exist measurable sets $\mathbb{Z}_{t} \subseteq \mathbb{Z}, t \in[0, T]$, such that:

(a) $P^{(\delta)}\left(t, z_{\delta}, t+u, \cdot\right) \Rightarrow P^{(0)}(t, z, t+u, \cdot)$ as $\delta \rightarrow 0$ for an arbitrary $z_{\delta} \rightarrow z \in \mathbb{Z}_{t}$ as $\delta \rightarrow 0$ if $0 \leq t<t+u \leq T$;

(b) $P^{(0)}\left(t, z, t+u, \mathbb{Z}_{t+u}\right)=1$ for all $z \in \mathbb{Z}_{t}$ if $0 \leq t<t+u \leq T$.

A typical example is given by the sets $\overline{\mathbb{Z}}_{t}=\varnothing$. In this case, condition $\mathbf{B}_{\mathbf{3}}(\mathbf{b})$ automatically holds. Another typical example is the one where $\mathbb{Z}_{t}=\mathbb{Y}_{t} \times \mathbb{X}$ and the sets $\overline{\mathbb{Y}}_{t}$ are at most countable. In this case, the assumption that the measures

$$
P^{(0)}(t, z, t+u, A \times \mathbb{X}), \quad A \in \mathcal{B}_{1},
$$

have no atoms implies condition $\mathbf{B}_{\mathbf{3}}(\mathbf{b})$.

The second condition asserts the weak convergence of the initial distributions to some distribution concentrated at the sets of convergence of the corresponding transition probabilities:

$\mathbf{B}_{\mathbf{4}}:$ (a) $P^{(\delta)}(\cdot) \Rightarrow P^{(0)}(\cdot)$ as $\delta \rightarrow 0$;

(b) $P^{(0)}\left(\mathbb{Z}_{0}\right)=1$, where $\mathbb{Z}_{0}$ is the set introduced in condition $\mathbf{B}_{\mathbf{3}}$.

A typical example is presented by an empty set $\overline{\mathbb{Z}}_{0}$. In this case, condition $\mathbf{B}_{4}(\mathbf{b})$ holds automatically. In the case, where $\mathbb{Z}_{0}=\mathbb{Y}_{0} \times \mathbb{X}$ and $\overline{\mathbb{Y}}_{0}$ is at most countable, the assumption that measure $P^{(0)}(A \times \mathbb{X}), A \in \mathcal{B}_{1}$, has no atoms implies condition $\mathbf{B}_{\mathbf{4}}(\mathbf{b})$.

Condition $\mathbf{B}_{4}$ holds, for example, if the initial distributions $P^{(\delta)}(A)=\chi_{A}\left(z_{0}\right), \delta \geq 0$, are concentrated at a point $z_{0} \in \mathbb{Z}_{0}$. This condition also holds if the initial distributions are such that $P^{(\delta)}(A)=\chi_{A}\left(z_{\delta}\right)$ for $\delta \geq 0$, where $z_{\delta} \rightarrow z_{0}$ as $\delta \rightarrow 0$ and $z_{0} \in \mathbb{Z}_{0}$.

The following theorem is the third main result of the present paper. It describes conditions for the convergence of reward functionals $\Phi\left(\mathcal{M}_{\max , T}^{(\delta)}\right)$.

Theorem 3. Let conditions $\mathbf{A}_{\mathbf{1}}, \mathbf{A}_{\mathbf{4}}, \mathbf{B}_{\mathbf{3}}, \mathbf{B}_{\mathbf{4}}, \mathbf{C}_{\mathbf{1}}$, and $\mathbf{C}_{\mathbf{2}}$ hold. Then

$$
\Phi\left(\mathcal{M}_{\max , T}^{(\delta)}\right) \rightarrow \Phi\left(\mathcal{M}_{\max , T}^{(0)}\right)<\infty \quad \text { as } \delta \rightarrow 0 .
$$

Proof of Theorem 3. Let $\Pi_{N}=\left\{0=t_{0, N}<t_{1, N}<\cdots<t_{N, N}=T\right\}$ be a sequence of partitions such that $d\left(\Pi_{N}\right) \rightarrow 0$ as $N \rightarrow \infty$.

Lemma 5. Let conditions $\mathbf{A}_{\mathbf{1}}, \mathbf{C}_{\mathbf{1}}$, and $\mathbf{C}_{\mathbf{2}}$ hold. Then

$$
\lim _{N \rightarrow \infty} \varlimsup_{\delta \rightarrow 0}\left(\Phi\left(\mathcal{M}_{\max , T}^{(\delta)}\right)-\Phi\left(\mathcal{M}_{\Pi_{N}, T}^{(\delta)}\right)\right)=0
$$

for an arbitrary sequence of partitions $\Pi_{N}$ such that $d\left(\Pi_{N}\right) \rightarrow 0$ as $N \rightarrow \infty$.

Proof of Lemma 5. This lemma follows directly from Theorem 1. Indeed, Theorem 1 implies that, under conditions $\mathbf{A}_{\mathbf{1}}, \mathbf{C}_{\mathbf{1}}$, and $\mathbf{C}_{\mathbf{2}}$, there exist constants $L_{3}, L_{4}<\infty$ such that the following skeleton approximation inequality holds for $\delta \leq \delta_{1}$ if $N$ is such that $d\left(\Pi_{N}\right) \leq c$ (the numbers $\delta_{1}$ and $c$ are defined in Theorem 1$)$ :

$$
\Phi\left(\mathcal{M}_{\max , T}^{(\delta)}\right)-\Phi\left(\mathcal{M}_{\Pi_{N}, T}^{(\delta)}\right) \leq L_{3} d\left(\Pi_{N}\right)+L_{4}\left(\Delta_{\beta}\left(Y^{(\delta)}(\cdot), d\left(\Pi_{N}\right), T\right)\right)^{\frac{\beta-\gamma}{\beta}} .
$$

This estimate implies relation (24).

The following lemma shows that the hypotheses of Theorem 3 imply the hypotheses of Theorem 2 for any partition $\Pi_{N}$ of the interval $[0, T]$. 
Lemma 6. Let conditions $\mathbf{A}_{\mathbf{1}}, \mathbf{A}_{\mathbf{4}}, \mathbf{B}_{\mathbf{3}}, \mathbf{B}_{\mathbf{4}}, \mathbf{C}_{\mathbf{1}}$, and $\mathbf{C}_{\mathbf{2}}$ hold. Then the hypotheses of Theorem 2 are satisfied for any partition $\Pi_{N}=\left\{0=t_{0}<t_{1}<\cdots<t_{N}=T\right\}$ of the interval $[0, T]$. Therefore

$$
\Phi\left(\mathcal{M}_{\Pi_{N}, T}^{(\delta)}\right) \rightarrow \Phi\left(\mathcal{M}_{\Pi_{N}, T}^{(0)}\right) \quad \text { as } \delta \rightarrow 0 .
$$

Proof. Conditions $\mathbf{A}_{\mathbf{1}}(\mathbf{c})$ and (d) imply that, for all $t \in[0, T], 0<s<\infty$, and $\delta \leq \delta_{0}$,

$$
\begin{aligned}
g^{(\delta)}(t, s) & \leq \int_{0}^{s}\left|\frac{\partial g^{(\delta)}(t, u)}{\partial u}\right| d u+g^{(\delta)}(t, 0) \\
& \leq \int_{0}^{s}\left(K_{3}+K_{4} u^{\gamma_{2}}\right) d u+K_{5} \\
& =K_{3} s+\frac{K_{4}}{\gamma_{2}+1} s^{\gamma_{2}+1}+K_{5} \leq K_{6}+K_{7} s^{\gamma},
\end{aligned}
$$

where $K_{6}=K_{3}+K_{4}+K_{5}$ and $K_{7}=K_{3}+K_{4}$.

Thus condition $\mathbf{A}_{\mathbf{2}}$ holds for any partition $\Pi_{N}$ of the interval $[0, T]$.

Condition $\mathbf{A}_{\mathbf{1}}(\mathbf{c})$ implies also that

$$
\begin{aligned}
\left|g^{(\delta)}\left(t, s^{\prime \prime}\right)-g^{(\delta)}\left(t, s^{\prime \prime}\right)\right| & \leq \int_{s^{\prime}}^{s^{\prime \prime}}\left|\frac{\partial g^{(\delta)}(t, u)}{\partial u}\right| d u \\
& \leq \int_{s^{\prime}}^{s^{\prime \prime}}\left(K_{3}+K_{4} u^{\gamma_{2}}\right) d u \leq\left(K_{3}+K_{4}\left(s^{\prime \prime}\right)^{\gamma}\right)\left(s^{\prime \prime}-s^{\prime}\right)
\end{aligned}
$$

for all $t \in[0, T], 0<s^{\prime}<s^{\prime \prime}<\infty$, and $\delta \leq \delta_{0}$,

Thus the following bound holds for the modulus of compactness in the uniform topology for pay-off functions:

$$
\sup _{s^{-} \leq s^{\prime} \leq s^{\prime \prime} \leq s^{\prime}+c \leq s^{+}}\left|g^{(\delta)}\left(t, s^{\prime}\right)-g^{(\delta)}\left(t, s^{\prime \prime}\right)\right| \leq\left(K_{3}+K_{4}\left(s^{+}\right)^{\gamma}\right) c
$$

for all $t \in[0, T], 0<s^{-}<s^{+}<\infty$, and $\delta \leq \delta_{0}$.

Relation (29) and condition $\mathbf{A}_{\mathbf{4}}$ imply that the hypotheses of the Ascoli-Arzelà theorem hold for the pay-off functions

$$
g^{(\delta)}(t, s), \quad s \in\left[s^{-}, s^{+}\right],
$$

for all $t \in[0, T]$ and $0<s^{-}<s^{+}<\infty$. Thus, for all $t \in[0, T]$, these functions converge uniformly, that is,

$$
\sup _{s^{-} \leq s \leq s^{+}}\left|g^{(\delta)}(t, s)-g^{(0)}(t, s)\right| \rightarrow 0 \quad \text { as } \delta \rightarrow 0 .
$$

Relation (30) implies that the condition on the locally uniform convergence $\mathbf{A}_{\mathbf{3}}$ holds for any partition $\Pi_{N}$ of the interval $[0, T]$ with the sets $\mathbb{S}_{t_{n}}=(0, \infty)$. In this case, the sets are such that $\mathbb{Y}_{t_{n}}=\ln \mathbb{S}_{t_{n}}=\mathbb{R}_{1}$ and therefore $\mathbb{Z}_{t_{n}}^{\prime}=\mathbb{Z}$.

If $\mathbb{Z}_{t_{n}}^{\prime}=\mathbb{Z}$ for all $t_{n} \in[0, T]$, then condition $\mathbf{B}_{\mathbf{3}}$ implies condition $\mathbf{B}_{\mathbf{1}}$ with the sets $\mathbb{Z}_{t_{n}}$ defined in condition $\mathbf{B}_{\mathbf{3}}$ for any partition $\Pi_{N}$ of the interval $[0, T]$. In its turn, condition $\mathbf{B}_{\mathbf{4}}$ implies that condition $\mathbf{B}_{\mathbf{2}}$ holds with the sets $\mathbb{Z}_{t_{n}}^{\prime}=\mathbb{Z}$ for any partition $\Pi_{N}$ of the interval $[0, T]$.

It remains to show that condition $\mathbf{C}_{\mathbf{1}}$ implies condition $\mathbf{C}_{\mathbf{3}}$. Condition $\mathbf{C}_{\boldsymbol{1}}$ implies that, for any constant $L_{12}<\infty$, one can choose $c=c\left(L_{12}\right)>0$ and then $\delta_{3}=\delta_{3}(c)<\delta_{0}$ such that

$$
\Delta_{\beta}\left(Y^{(\delta)}(\cdot), c, T\right) \leq L_{12}
$$

for all $\delta \leq \delta_{3}$. 
Consider an arbitrary integer $0 \leq n<N$ and the uniform partition

$$
t_{n}=u_{0, m}<\cdots<u_{m, m}=t_{n+1}
$$

of the interval $\left[t_{n}, t_{n+1}\right]$ by points $u_{k, m}=\left(t_{n+1}-t_{n}\right) k / m$. Using inequality (31) and the Markov property of the process $Z^{(\delta)}(t)$ we get

$$
\begin{aligned}
& \mathrm{E}_{z, t_{n}}\left(e^{\beta\left|Y^{(\delta)}\left(u_{k, m}\right)-Y^{(\delta)}\left(u_{0, m}\right)\right|}-1\right) \\
& \leq \mathrm{E}_{z, t_{n}} e^{\beta\left|Y^{(\delta)}\left(u_{k-1, m}\right)-Y^{(\delta)}\left(u_{0, m}\right)\right|} e^{\beta\left|Y^{(\delta)}\left(u_{k, m}\right)-Y^{(\delta)}\left(u_{k-1, m}\right)\right|}-1 \\
&= \mathrm{E}_{z, t_{n}}\left(\left(e^{\beta\left|Y^{(\delta)}\left(u_{k-1, m}\right)-Y^{(\delta)}\left(u_{0, m}\right)\right|}-1\right) e^{\beta\left|Y^{(\delta)}\left(u_{k, m}\right)-Y^{(\delta)}\left(u_{k-1, m}\right)\right|}\right. \\
&= \mathrm{E}_{z, t_{n}}\left\{\left(e^{\beta\left|Y^{(\delta)}\left(u_{k, m}\right)-Y^{(\delta)}\left(u_{k-1, m}\right)\right|}-1\right)\right. \\
&\left.\times \mathrm{E}\left\{\left(e^{\beta\left|Y^{(\delta)}\left(u_{k, m}\right)-Y^{(\delta)}\left(u_{k-1, m}\right)\right|}-1+1\right) / Z^{(\delta)}\left(u_{k-1, m}\right)\right\}\right\} \\
&+\mathrm{E}_{z, t_{n}}\left\{\mathrm{E}\left\{\left(e^{\beta\left|Y^{(\delta)}\left(u_{k, m}\right)-Y^{(\delta)}\left(u_{k-1, m}\right)\right|}-1\right) / Z^{(\delta)}\left(u_{k-1, m}\right)\right\}\right\} \\
& \leq \mathrm{E}_{z, t_{n}}\left(e^{\beta\left|Y^{(\delta)}\left(u_{k-1, m}\right)-Y^{(\delta)}\left(u_{0, m}\right)\right|}-1\right)\left(L_{12}+1\right)+L_{12}
\end{aligned}
$$

for $\delta \leq \delta_{3}, m=\left[\left(t_{n+1}-t_{n}\right) / c\right]+1$ (in this case $\left.\left(t_{n+1}-t_{n}\right) / m \leq c\right), z \in \mathbb{Z}$, and $k=1, \ldots, m$.

Finally, we get for $\delta \leq \delta_{3}$ and $z \in \mathbb{Z}$,

$$
\begin{aligned}
\mathrm{E}_{z, t_{n}} & \left(e^{\beta\left|Y^{(\delta)}\left(t_{n+1}\right)-Y^{(\delta)}\left(t_{n}\right)\right|}-1\right) \\
& =\mathrm{E}_{z, t_{n}}\left(e^{\beta\left|Y^{(\delta)}\left(u_{m, m}\right)-Y^{(\delta)}\left(u_{0, m}\right)\right|}-1\right) \\
& \leq\left(L_{12}+1\right)^{m}+L_{12} \sum_{k=0}^{m-1}\left(L_{12}+1\right)^{k}=2\left(L_{12}+1\right)^{m}-1<\infty .
\end{aligned}
$$

Thus condition $\mathbf{C}_{\mathbf{3}}$ holds. Finally, condition $\mathbf{C}_{\mathbf{2}}$ is equivalent to condition $\mathbf{C}_{\mathbf{4}}$. The proof is complete.

Lemmas 5 and 6 allow us to make the final step in the proof of Theorem 3. We employ the following obvious inequality:

$$
\begin{aligned}
& \left|\Phi\left(\mathcal{M}_{\max , T}^{(\delta)}\right)-\Phi\left(\mathcal{M}_{\max , T}^{(0)}\right)\right| \\
& \leq\left|\Phi\left(\mathcal{M}_{\max , T}^{(\delta)}\right)-\Phi\left(\mathcal{M}_{\Pi_{N}, T}^{(\delta)}\right)\right|+\left|\Phi\left(\mathcal{M}_{\max , T}^{(0)}\right)-\Phi\left(\mathcal{M}_{\Pi_{N}, T}^{(0)}\right)\right| \\
& \quad+\left|\Phi\left(\mathcal{M}_{\Pi_{N}, T}^{(\delta)}\right)-\Phi\left(\mathcal{M}_{\Pi_{N}, T}^{(0)}\right)\right| .
\end{aligned}
$$

Here $\Pi_{N}$ is an arbitrary partition.

Using the latter inequality and relation (26) we get

$$
\begin{aligned}
\varlimsup_{\delta \rightarrow 0} & \left|\Phi\left(\mathcal{M}_{\max , T}^{(\delta)}\right)-\Phi\left(\mathcal{M}_{\max , T}^{(0)}\right)\right| \\
& \leq \varlimsup_{\delta \rightarrow 0}\left|\Phi\left(\mathcal{M}_{\max , T}^{(\delta)}\right)-\Phi\left(\mathcal{M}_{\Pi_{N}, T}^{(\delta)}\right)\right|+\left|\Phi\left(\mathcal{M}_{\max , T}^{(0)}\right)-\Phi\left(\mathcal{M}_{\Pi_{N}, T}^{(0)}\right)\right|
\end{aligned}
$$

for any partition $\Pi_{N}$.

Finally, relation (24) implies that the expression on the right hand side of (35) can be made as small as necessary by choosing a partition $\Pi_{N}$ whose diameter $d\left(\Pi_{N}\right)$ is sufficiently small (note that the relation $\delta \rightarrow 0$ includes the case of $\delta=0$ ). 
This proves the asymptotic relation (23) and completes the proof of Theorem 3.

\section{Compactness COnditions FOR LOG-PRICE PROCESSES}

First we make several useful remarks concerning the evaluation of the exponential modulus of compactness $\Delta_{\beta}\left(Y^{(\delta)}(\cdot), c, T\right)$.

The exponential modulus of compactness $\Delta_{\beta}\left(Y^{(\delta)}(\cdot), c, T\right)$ admits the following representation:

$$
\begin{aligned}
\Delta_{\beta}\left(Y^{(\delta)}(\cdot), c, T\right) & =\sup _{0 \leq t \leq t+u \leq t+c \leq T} \sup _{z \in \mathbb{Z}}\left(\mathrm{E}_{z, t} e^{\beta\left|Y^{(\delta)}(t+u)-Y^{(\delta)}(t)\right|}-1\right) \\
& =\sup _{0 \leq t \leq t+u \leq t+c \leq T} \sup _{z \in \mathbb{Z}} \beta \int_{0}^{\infty} e^{\beta y} \mathrm{P}_{z, t}\left\{\left|Y^{(\delta)}(t+u)-Y^{(\delta)}(t)\right|>y\right\} d y
\end{aligned}
$$

This representation shows that the compactness condition $\mathbf{C}_{\mathbf{1}}$ can effectively be used if the tail probabilities for increments $\left|Y^{(\delta)}(t+u)-Y^{(\delta)}(t)\right|$ are given explicitly or can effectively be estimated.

Condition $\mathbf{C}_{\mathbf{1}}$ on the exponential moment compactness can also be compared with the traditional condition on the compactness in the J-topology for Markov type càdlàg processes. The modulus of J-compactness is defined by

$$
\Delta\left(Y^{(\delta)}(\cdot), h, c, T\right)=\sup _{0 \leq t \leq t+u \leq t+c \leq T} \sup _{z \in \mathbb{Z}} \mathrm{P}_{z, t}\left\{\left|Y^{(\delta)}(t+u)-Y^{(\delta)}(t)\right| \geq h\right\}
$$

The following condition on the J-compactness plays a key role in functional limit theorems for Markov type càdlàg processes:

$\mathbf{C}_{\mathbf{5}}: \lim _{c \rightarrow 0} \varlimsup_{\overline{\lim \rightarrow 0}} \Delta\left(Y^{(\delta)}(\cdot), h, c, T\right)=0, h>0$.

We also introduce an index that is the maximum of the moment generating functions for increments of the log-price process $Y^{(\delta)}(t)$ :

$$
\Xi_{\beta}\left(Y^{(\delta)}(\cdot), T\right)=\sup _{0 \leq t \leq t+u \leq T} \sup _{z \in \mathbb{Z}} \mathrm{E}_{z, t} e^{\beta\left(Y^{(\delta)}(t+u)-Y^{(\delta)}(t)\right)} .
$$

The following condition is stated in terms of the moment generating functions. Its advantage is that it can effectively be verified in many useful cases:

$\mathbf{C}_{\mathbf{6}}: \varlimsup_{\overline{\lim \rightarrow 0}} \Xi_{ \pm \beta^{\prime}}\left(Y^{(\delta)}(\cdot), T\right)<\infty$ for some $\beta^{\prime}>\beta$, where $\beta$ is the parameter involved in condition $\mathbf{C}_{\mathbf{1}}$.

Lemma 7. Conditions $\mathbf{C}_{\mathbf{5}}$ and $\mathbf{C}_{\mathbf{6}}$ imply condition $\mathbf{C}_{\mathbf{1}}$.

Proof of Lemma 7. Using Hölder's inequality we get

$$
\begin{aligned}
\mathrm{E}_{z, t} & e^{\beta\left|Y^{(\delta)}(t+u)-Y^{(\delta)}(t)\right|}-1 \\
\leq & \left(e^{\beta h}-1\right)+\mathrm{E}_{z, t} e^{\beta\left|Y^{(\delta)}(t+u)-Y^{(\delta)}(t)\right|} \chi\left(\left|Y^{(\delta)}(t+u)-Y^{(\delta)}(t)\right| \geq h\right) \\
\leq & \left(e^{\beta h}-1\right) \\
& \quad+\left(\mathrm{E}_{z, t} e^{\beta^{\prime}\left|Y^{(\delta)}(t+u)-Y^{(\delta)}(t)\right|}\right)^{\frac{\beta}{\beta^{\prime}}} \mathrm{P}_{z, t}\left\{\left|Y^{(\delta)}(t+u)-Y^{(\delta)}(t)\right| \geq h\right\}
\end{aligned}
$$

for all $0 \leq t \leq t+u \leq T$ and $z \in \mathbb{Z}$.

The following inequality relates the exponential moment modulus of compactness to the modulus of J-compactness:

$$
\Delta_{\beta}\left(Y^{(\delta)}(\cdot), c, T\right) \leq\left(e^{\beta h}-1\right)+\left(\Delta_{\beta^{\prime}}\left(Y^{(\delta)}(\cdot), c, T\right)+1\right)^{\frac{\beta}{\beta^{\prime}}} \Delta\left(Y^{(\delta)}(\cdot), h, c, T\right) .
$$

Inequality (37) follows readily from (36). 
Further

$$
\begin{aligned}
\mathrm{E}_{z, t} e^{\beta^{\prime}\left|Y^{(\delta)}(t+u)-Y^{(\delta)}(t)\right|}= & \mathrm{E}_{z, t} e^{\beta^{\prime}\left(Y^{(\delta)}(t+u)-Y^{(\delta)}(t)\right)} \chi\left(Y^{(\delta)}(t+u) \geq Y^{(\delta)}(t)\right) \\
& +\mathrm{E}_{z, t} e^{-\beta^{\prime}\left(Y^{(\delta)}(t+u)-Y^{(\delta)}(t)\right)} \chi\left(Y^{(\delta)}(t+u)<Y^{(\delta)}(t)\right) \\
\leq & \mathrm{E}_{z, t} e^{\beta^{\prime}\left(Y^{(\delta)}(t+u)-Y^{(\delta)}(t)\right)}+\mathrm{E}_{z, t} e^{-\beta^{\prime}\left(Y^{(\delta)}(t+u)-Y^{(\delta)}(t)\right)}
\end{aligned}
$$

for all $0 \leq t \leq t+u \leq T$ and $z \in \mathbb{Z}$.

Bound (38) implies that

$$
\Delta_{\beta^{\prime}}\left(Y^{(\delta)}(\cdot), c, T\right)+1 \leq \Xi_{\beta^{\prime}}\left(Y^{(\delta)}(\cdot), T\right)+\Xi_{-\beta^{\prime}}\left(Y^{(\delta)}(\cdot), T\right) .
$$

Relations (37) and inequality (39) prove Lemma 7.

\section{EXAMPLES}

In this section, Theorems 1-3 are applied to some important cases.

Consider a model without modulation. This means that the phase space $\mathbb{X}=\left\{x_{0}\right\}$ degenerates to a single point, while the $\log$-price $Y^{(\delta)}(t), t \geq 0$, is a càdlàg process with independent increments.

For the sake of simplicity, we also assume that the initial state of this process is a constant, that is, $y_{\delta}=Y^{(\delta)}(0)$.

Then the $\log$-price $Y^{(\delta)}(t)$ is a càdlàg Markov process whose transition probabilities are related to the distributions $P^{(\delta)}(t, t+u, A)$ of increments of this process as follows:

$$
\begin{aligned}
P^{(\delta)}(t, y, t+u, A) & =P^{(\delta)}(t, t+u, A-y) \\
& =\mathrm{P}\left\{y+Y^{(\delta)}(t+u)-Y^{(\delta)}(t) \in A\right\} .
\end{aligned}
$$

We assume the following standard condition of the weak convergence for the distributions of increments of log-price processes:

$\mathbf{D}_{\mathbf{1}}: P^{(\delta)}(t, t+u, \cdot) \Rightarrow P^{(0)}(t, t+u, \cdot)$ as $\delta \rightarrow 0,0 \leq t \leq t+u \leq T$.

Representation (40) implies that condition $\mathbf{B}_{\mathbf{3}}$ holds with the sets $\mathbb{Y}_{t}=\mathbb{R}_{1}, t \in[0, T]$. In other words, the distributions of increments of the processes $Y^{(\delta)}(t)$ locally uniformly weakly converge if condition $\mathbf{D}_{\mathbf{1}}$ holds. Thus condition $\mathbf{B}_{\mathbf{3}}$ with the sets $\mathbb{Y}_{t}=\mathbb{R}_{1}$ is, in fact, equivalent to the standard condition of the weak convergence for processes with independent increments,.

In this case, the modulus $\Delta\left(Y^{(\delta)}(\cdot), h, c, T\right)$ of $J$-compactness becomes of the following form:

$$
\Delta\left(Y^{(\delta)}(\cdot), h, c, T\right)=\sup _{0 \leq t \leq t+u \leq t+c \leq T} \mathrm{P}\left\{\left|Y^{(\delta)}(t+u)-Y^{(\delta)}(t)\right| \geq h\right\} .
$$

Thus condition $\mathbf{C}_{\mathbf{5}}$ is reduced to the standard condition of $J$-compactness for log-price processes:

$\mathbf{D}_{\mathbf{2}}: \varlimsup_{c \rightarrow 0} \lim _{\delta \rightarrow 0} \Delta\left(Y^{(\delta)}(t), h, c, T\right)=0, h>0$.

Note that conditions $\mathbf{D}_{\mathbf{1}}$ and $\mathbf{D}_{\mathbf{2}}$ imply the $J$-convergence of log-prices

$$
Y^{(\delta)}(t), \quad t \in[0, T]
$$

to the process $Y^{(0)}(t), t \in[0, T]$, as $\delta \rightarrow 0$. The stochastic continuity of the limit process follows from conditions $\mathbf{D}_{\mathbf{1}}$ and $\mathbf{D}_{\mathbf{2}}$.

In this case, the index $\Xi_{\beta}\left(Y^{(\delta)}(\cdot), T\right)$ becomes of a simpler form:

$$
\Xi_{\beta}\left(Y^{(\delta)}(\cdot), T\right)=\sup _{0 \leq t \leq t+u \leq T} \mathrm{E} e^{\beta\left(Y^{(\delta)}(t+u)-Y^{(\delta)}(t)\right)} .
$$


Therefore, condition $\mathbf{C}_{6}$ transforms to

$\mathbf{D}_{\mathbf{3}}: \overline{\lim }_{\delta \rightarrow 0} \Xi_{ \pm \beta^{\prime}}\left(Y^{(\delta)}(\cdot), T\right)<\infty$ for some $\beta^{\prime}>\beta$, where $\beta$ is the parameter defined in condition $\mathbf{C}_{\mathbf{1}}$.

According to Lemma 7 conditions $\mathbf{D}_{\mathbf{1}}$ and $\mathbf{D}_{\mathbf{2}}$ imply condition $\mathbf{C}_{\mathbf{1}}$. In its turn, $\mathbf{B}_{\mathbf{4}}$ is reduced in this case to

$\mathbf{D}_{4}: \lim _{\delta \rightarrow 0} y_{\delta}=y_{0}$.

Note that $y_{0}$ can be an arbitrary real number, since $\mathbb{Y}_{0}=\mathbb{R}_{1}$. Obviously, condition $\mathbf{D}_{\mathbf{4}}$ implies condition $\mathbf{C}_{\mathbf{4}}$.

Collecting the remarks above, we conclude that Theorem 3 holds for the exponential price processes with independent increments $S^{(\delta)}(t)=e^{Y^{(\delta)}(t)}$ if conditions $\mathbf{A}_{\mathbf{1}}, \mathbf{A}_{\mathbf{4}}$, and $\mathbf{D}_{\mathbf{1}}-\mathbf{D}_{\mathbf{4}}$ are satisfied.

The skeleton approximations $Y^{(\delta)}(t)=Y^{(0)}([t / \delta]), t \geq 0$, for a stochastically continuous càdlàg log-price process $Y^{(0)}(t), t \geq 0$, with independent increments present an example of the model introduced above. In this case, conditions $\mathbf{D}_{\mathbf{1}}$ and $\mathbf{D}_{\mathbf{2}}$ automatically hold.

Condition $\mathbf{D}_{\mathbf{3}}$ follows from

$\mathbf{D}_{5}: \Xi_{ \pm \beta^{\prime}}\left(Y^{(0)}(\cdot), T\right)<\infty$ for some $\beta^{\prime}>\beta$, where $\beta$ is the parameter defined in condition $\mathbf{C}_{\mathbf{1}}$.

Thus, Theorem 3 holds for the exponential price processes

$$
S^{(\delta)}(t)=e^{Y^{(0)}([t / \delta])}, \quad t \in[0, T],
$$

if conditions $\mathbf{A}_{1}, \mathbf{A}_{\mathbf{4}}$, and $\mathbf{D}_{5}$ hold.

It is worthwhile mentioning that Theorem 1 proved in the first part of this paper, see [1, yields a stronger result expressed in terms of explicit estimates for the accuracy of skeleton approximations of reward functions.

Note also that the optimal expected rewards for the skeleton price processes

$$
S^{(\delta)}(t)=e^{Y^{(0)}([t / \delta])}
$$

can be estimated with the help of the Monte Carlo method. The corresponding algorithms are described, for example, in Silvestrov, Galochkin and Sibirtsev [13, Silvestrov, Galochkin, and Malyarenko [12, Kukush and Silvestrov [8, [9], Jönsson, Kukush, and Silvestrov [5], [], Jönsson [4].

A combination of these estimates based on the Monte Carlo method with the skeleton approximations described above yields an effective approximation method for the optimal expected rewards for American type options with non-standard payoffs.

Now we consider a model, where the log-prices $Y^{(\delta)}(t), t \geq 0$, are càdlàg Lévy processes for all $\delta \geq 0$. The Lévy-Khintchine representation of $Y^{(\delta)}(t)$ is given by

$$
\begin{aligned}
\varphi_{t}^{(\delta)}(v) & =\operatorname{E} \exp \left\{i v\left(Y^{(\delta)}(t)-y_{\delta}\right)\right\} \\
& =\exp \left\{i t a_{\delta} v-\frac{1}{2} t b_{\delta}^{2} v^{2}+t \int_{\mathbb{R}_{1}}\left(e^{i v s}-1-\frac{i v s}{1+s^{2}}\right) \Pi_{\delta}(d s)\right\}, \\
v & \in \mathbb{R}_{1}, \quad t \geq 0,
\end{aligned}
$$

where

(a) $a_{\delta} \in \mathbb{R}_{1}$;

(b) $b_{\delta} \geq 0$;

(c) $\Pi_{\delta}(A)$ is a measure on $\mathbb{R}_{1}$ such that

$$
\int_{|s| \leq 1} s^{2} \Pi_{\delta}(d s)<\infty ;
$$


(d) $y_{\delta}=Y^{(\delta)}(0)$ is an initial state of this process, assumed for simplicity to be a non-random constant.

We further assume the following standard condition, which is necessary and sufficient for the weak convergence of increments of the processes $Y^{(\delta)}(t)$ :

$\mathbf{E}_{\mathbf{1}}:$ (a) $\lim _{\delta \rightarrow 0} a_{\delta}=a_{0}$;

(b) $\varlimsup_{\varepsilon \rightarrow 0} \lim _{\delta \rightarrow 0}\left|b_{0}^{2}-b_{\delta}^{2}-\int_{|s| \leq \varepsilon} s^{2} \Pi_{\delta}(d s)\right|=0$;

(c) $\lim _{\delta \rightarrow 0} \int_{\mathbb{R}_{1}} f(s) \Pi_{\delta}(d s)=\int_{\mathbb{R}_{1}} f(s) \Pi_{0}(d s)$ for all continuous bounded functions $f(s)$ vanishing in some neighborhood of the origin.

It is well known in this case that condition $\mathbf{E}_{\mathbf{1}}$ implies $\mathbf{D}_{\mathbf{1}}$ and $\mathbf{D}_{\mathbf{2}}$ (see, for example, Skorokhod [15]).

Also is known that the moment generating function $\mathrm{E} \exp \left\{w Y^{(\delta)}(t)\right\}=\psi_{t}^{(\delta)}(w)$ exists for a given $w \in \mathbb{R}_{1}$ if and only if

$$
\int_{|s|>1} e^{w s} \Pi_{\delta}(d s)<\infty
$$

Moreover, $\psi_{t}^{(\delta)}(w)$ can be expressed in terms of the corresponding characteristic function, namely $\psi_{t}^{(\delta)}(w)=\varphi^{(\delta)}(w / i)$.

Taking into account the above reasoning we conclude that condition $\mathbf{D}_{\mathbf{3}}$ follows from condition $\mathbf{D}_{5}$ and

$\mathbf{E}_{\mathbf{2}}: \varlimsup_{\delta \rightarrow 0} \int_{|s|>1} e^{\beta^{\prime}|s|} \Pi_{\delta}(d s)<\infty$, where $\beta^{\prime}$ is the parameter defined in condition $\mathbf{C}_{6}$.

Therefore Theorem 3 holds for the exponential Lévy price processes introduced above if conditions $\mathbf{A}_{\mathbf{1}}, \mathbf{A}_{\mathbf{4}}, \mathbf{D}_{\mathbf{4}}$, and $\mathbf{E}_{\mathbf{1}}-\mathbf{E}_{\mathbf{2}}$ hold.

For the skeleton approximations $S^{(\delta)}(t)=e^{Y^{(0)}([t / \delta])}$, we note that condition $\mathbf{D}_{\mathbf{5}}$ follows from

$\mathbf{E}_{\mathbf{3}}: \int_{|s|>1} e^{\beta^{\prime}|s|} \Pi_{0}(d s)<\infty$, where $\beta^{\prime}$ is the parameter defined in condition $\mathbf{C}_{\mathbf{6}}$.

Theorem 3 follows for the exponential price processes $S^{(\delta)}(t)=e^{Y^{(0)}([t / \delta])}$ from conditions $\mathbf{A}_{\mathbf{1}}, \mathbf{A}_{\mathbf{4}}$, and $\mathbf{E}_{\mathbf{3}}$.

This result can be generalized to the model of time non-homogeneous, stochastically continuous, càdlàg exponential price processes with independent increments.

Consider a price process $S^{(\delta)}(t)=e^{Y^{(\delta)}(t)}$, which can be referred to as an exponential process with independent increments modulated by a semi-Markov stochastic index process. In this model, the $\log$-price $Y^{(\delta)}(t)$ is represented as follows:

$$
Y^{(\delta)}(t)=y_{\delta}+\sum_{n=0}^{N^{(\delta)}(t)-1} Y_{n, X_{n}^{(\delta)}}^{(\delta)}\left(T_{n+1}^{(\delta)}-T_{n}^{(\delta)}\right)+Y_{N^{(\delta)}(t), X^{(\delta)}(t)}^{(\delta)}\left(T^{(\delta)}(t)\right), \quad t \geq 0
$$

where

(e) $X^{(\delta)}(t), t \geq 0$, is a continuous on the right semi-Markov process with a finite set of states $\mathbb{X}=\{1, \ldots, m\}$ and transition probabilities $Q_{i j}^{(\delta)}(t), t \geq 0, i, j \in \mathbb{X}$;

(f) $N^{(\delta)}(t)$ is the number of jumps for the semi-Markov process $X^{(\delta)}(u)$ in the interval $[0, t]$;

(g) $X_{n}^{(\delta)}, n=0,1, \ldots$, are the states of the semi-Markov process $X^{(\delta)}(t)$ at sequential moments of jumps;

(h) $T_{n}^{(\delta)}, n=0,1, \ldots$, are the sequential moments of jumps of the semi-Markov process $X^{(\delta)}(t)$; 
(i) $T^{(\delta)}(t)=t-T_{N^{(\delta)}(t)}^{(\delta)}$ is the time between moment $t$ and the moment of the last jump of process $X^{(\delta)}(u)$ before $t$;

(j) $Y_{n, i}^{(\delta)}(t), t \geq 0$, for all $n=0,1, \ldots$ and $i \in \mathbb{X}$, is a real-valued càdlàg process with independent increments with an initial state $Y_{n, i}^{(\delta)}(0)=0$ and the distributions of increments

$$
P_{i}^{(\delta)}(t, t+s, A)=\mathrm{P}\left\{Y_{n, i}^{(\delta)}(t+s)-Y_{n, i}^{(\delta)}(t) \in A\right\},
$$

which do not depend on $n=0,1, \ldots$;

(k) the processes $Y_{n, i}^{(\delta)}(t), t \geq 0, n=0,1, \ldots, i \in \mathbb{X}$, and the process $X^{(\delta)}(t), t \geq 0$, are mutually independent.

We assume, for simplicity, that

(l) the initial state of the log-price process

$$
Y^{(\delta)}(0)=y_{0} \in \mathbb{R}_{1}
$$

and the semi-Markov index $X^{(\delta)}(0)=i_{0} \in \mathbb{X}$ are constants, and also that

(m) the semi-Markov index has no instant transition, that is, $Q_{i j}^{(\delta)}(0)=0, i, j \in \mathbb{X}$.

In this case, the two-component process $\left(Y^{(\delta)}(t), X^{(\delta)}(t)\right)$ is not Markovian. In contrast, $\tilde{X}^{(\delta)}(t)=\left(X^{(\delta)}(t), T^{(\delta)}(t)\right)$ is a homogeneous Markov process with the phase space $\tilde{\mathbb{X}}=\{1, \ldots, m\} \times[0, \infty)$. This process can be interpreted as a stochastic index modulating the log-price process $Y^{(\delta)}(t)$. The phase space of this process is a Polish space equipped with the standard metric $\rho((i, s),(j, t))=\sqrt{\chi(i \neq j)+|s-t|^{2}}$.

Then $\tilde{Z}^{(\delta)}(t)=\left(Y^{(\delta)}(t), \tilde{X}^{(\delta)}(t)\right), t \geq 0$, is a homogeneous Markov process. This also holds if $Y_{n, i}^{(\delta)}(t), t \geq 0$, are time non-homogeneous processes with independent increments.

The results formulated above for exponential processes with independent increments can be generalized to the model introduced here.

The transition probabilities of the semi-Markov process $X^{(\delta)}(t)$ can always be represented in the following form:

$$
Q_{i j}^{(\delta)}(t)=p_{i j}^{(\delta)} F_{i j}^{(\delta)}(t), \quad t \geq 0, i, j \in \mathbb{X},
$$

where $p_{i j}^{(\delta)}$ are the transition probabilities of the imbedded Markov chain $X_{n}^{(\delta)}$, while $F_{i j}^{(\delta)}(t)$ are the distributions of inter-jump times for this process conditioned on the states before and after jumps.

In this case, one should first assume the following condition of the weak convergence of transition probabilities for the stochastic semi-Markov indices:

$\mathbf{F}_{\mathbf{1}}:$ (a) $p_{i j}^{(\delta)} \rightarrow p_{i j}^{(0)}$ as $\delta \rightarrow 0, i, j \in \mathbb{X}$

(b) $F_{i j}^{(\delta)}(\cdot) \Rightarrow F_{i j}^{(0)}(\cdot)$ as $\delta \rightarrow 0, i, j \in \mathbb{X}$, where the limit distribution functions are continuous for all $i, j \in \mathbb{X}$.

Some further conditions should also be imposed on the log-price stochastic processes $Y_{n, i}^{(\delta)}(t)$, namely:

$\mathbf{F}_{\mathbf{2}}$ : Conditions $\mathbf{D}_{\mathbf{1}}-\mathbf{D}_{\mathbf{3}}$ hold for the processes $Y_{0, i}^{(\delta)}(t), t \in[0, T]$, for all $i \in \mathbb{X}$.

Let $F_{i}^{(\delta)}(t)$ be the distributions of sojourn times, that is,

$$
F_{i}^{(\delta)}(t)=\sum_{j \in \mathbb{X}} p_{i j}^{(\delta)} F_{i j}^{(\delta)}(t)
$$

and let $\tilde{\mathbb{Y}}=\left\{(i, t): F_{i}^{(0)}(t)<1, i \in \mathbb{X}\right\}$. 
Conditions $\mathbf{F}_{\mathbf{1}}$ and $\mathbf{F}_{\mathbf{2}}$ imply condition $\mathbf{B}_{\mathbf{1}}$ for the processes $\tilde{Z}^{(\delta)}(t), t \in[0, T]$, where $\mathbb{Z}_{t}=\mathbb{R}_{1} \times \tilde{\mathbb{Y}}, 0 \leq t \leq T$. Moreover conditions $\mathbf{C}_{\mathbf{5}}-\mathbf{C}_{\mathbf{6}}$ follow from $\mathbf{F}_{\mathbf{1}}$ and $\mathbf{F}_{\mathbf{2}}$.

The corresponding proofs can be found in Stenberg [16]. The proofs in Stenberg [16] are based on some estimates for the corresponding indices related to the log-price processes under a fixed trajectory of the semi-Markov index, the integration of these estimates with respect to the measure generated by the semi-Markov index in the space of its trajectories, and some estimates for the number of jumps of this index in the finite interval $[0, T]$.

Below we present a sketch of a corresponding proof related to condition $\mathbf{D}_{\mathbf{3}}$.

If condition $\mathbf{D}_{\mathbf{3}}$ holds for all processes $Y_{0, i}^{(\delta)}(t), t \in[0, T]$, and all $i \in \mathbb{X}$, then there exists a number $\delta_{4}>0$ such that

$$
\Xi_{ \pm \beta^{\prime}}(T)=\sup _{\delta \leq \delta_{4}} \max _{i \in \mathbb{X}} \Xi_{ \pm \beta^{\prime}}\left(Y_{0, i}^{(\delta)}(\cdot), T\right)<\infty .
$$

Condition $\mathbf{F}_{1}$ implies that for all $\alpha>0$ there exist $\delta_{5}=\delta_{5}(\alpha)>0$ such that

$$
\Upsilon(\alpha, T)=\sup _{\delta \leq \delta_{5}} \mathrm{E} e^{\alpha N^{(\delta)}(T)}<\infty .
$$

According to assumptions $(\mathrm{e})-(\mathrm{j})$, the $\log$-price $Y^{(\delta)}(t)$ is a process with independent increments conditionally independent with respect to the modulating stochastic semiMarkov index $X^{(\delta)}(t)$. Using (43)-(44), one can prove an estimate for

$$
\delta \leq \delta_{6}=\delta_{4} \wedge \delta_{5}\left(\ln \Xi_{ \pm \beta^{\prime}}(T)\right)
$$

and $0 \leq t \leq t+u \leq T$, namely

$$
\begin{aligned}
& \mathrm{E} e^{ \pm \beta^{\prime}\left(Y^{(\delta)}(t+u)-Y^{(\delta)}(t)\right)} \\
& =\mathrm{E} \sum_{n=0}^{\infty} \sum_{i_{1}, \ldots, i_{n} \in \mathbb{X}} \int_{0<t_{1}<\cdots<t_{n} \leq T} \mathrm{E}\left\{e^{ \pm \beta^{\prime}\left(Y^{(\delta)}(t+u)-Y^{(\delta)}(t)\right)} / \Lambda(\delta, n, T)\right\} \\
& \times \mathrm{P}\{\Lambda(\delta, n, T)\} \\
& \leq \sum_{n=0}^{\infty} \Xi_{ \pm \beta^{\prime}}(T)^{n+1} \mathrm{P}\left\{N^{(\delta)}(T)=n\right\}=\Xi_{ \pm \beta^{\prime}}(T) \Upsilon\left(\ln \left(\Xi_{ \pm \beta^{\prime}}(T)\right), T\right)<\infty
\end{aligned}
$$

where

$$
\Lambda(\delta, n, T)=\left\{X_{k}^{(\delta)}=i_{k}, T_{k}^{(\delta)} \in d t_{k}, k=1, \ldots, n, N^{(\delta)}(T)=n\right\} .
$$

Relation (45) obviously implies condition $\mathbf{D}_{\mathbf{3}}$ for processes $Y^{(\delta)}(t)$.

Note also that assumption $(\mathrm{k})$ automatically implies that conditions $\mathbf{B}_{\mathbf{4}}$ and $\mathbf{C}_{\mathbf{4}}$ hold for all initial states $y_{0}$ and $i_{0}$.

Collecting together the above remarks, one can conclude that Theorem 3 holds for the exponential price processes with independent increments modulated by the semi-Markov stochastic indices introduced above, provided conditions $\mathbf{A}_{\mathbf{1}}, \mathbf{A}_{\mathbf{4}}$, and $\mathbf{F}_{\mathbf{1}}-\mathbf{F}_{\mathbf{2}}$ hold.

In particular, Theorem 3 holds for the exponential Lévy price processes modulated by semi-Markov stochastic indices introduced above if conditions $\mathbf{A}_{\mathbf{1}}, \mathbf{A}_{\mathbf{4}}, \mathbf{F}_{\mathbf{1}}$ as well as conditions $\mathbf{E}_{\mathbf{1}}-\mathbf{E}_{\mathbf{2}}$ hold for the Lévy processes $Y_{0, i}^{(\delta)}(t), t \in[0, T], i \in \mathbb{X}$.

In conclusion, consider the case where the log-price processes $Y_{n, i}^{(\delta)}(t)$ are of the trinomial type. This means that $Y_{n, i}^{(\delta)}(t), \delta>0$, is represented in the following form:

$$
Y_{n, i}^{(\delta)}(t)=\sum_{1 \leq k \leq[t / \delta]} Y_{n, i, k}^{(\delta)}, \quad t \geq 0
$$


where $Y_{n, i, k}^{(\delta)}, k=1,2, \ldots, i \in \mathbb{X}, n=0,1, \ldots$, are independent random variables that take only three values, namely $u_{i}^{(\delta)}>0,0$, and $-u_{i}^{(\delta)}$ with the probabilities $p_{i}^{(\delta)}, r_{i}^{(\delta)}$ and $q_{i}^{(\delta)}$, respectively, where $p_{i}^{(\delta)}+r_{i}^{(\delta)}+q_{i}^{(\delta)}=1$.

Then the limit log-price processes $Y_{n, i}^{(0)}(t)$ are of the following form:

$$
Y_{n, i}^{(\delta)}(t)=\mu_{i} t+\sigma_{i} W_{n, i}(t), \quad t \geq 0
$$

where $W_{n, i}(t), t \geq 0$, are mutually independent for all $i \in \mathbb{X}$ and $n=0,1, \ldots$ standard Wiener processes.

In this case, $S^{(0)}(t)=\exp \left\{Y^{(0)}(t)\right\}$ is a geometric Brownian motion modulated by a semi-Markov stochastic index $X^{(0)}(t)$.

It is also natural to assume that the stochastic index $X^{(\delta)}(t)$ can jump only at the moments $\delta, 2 \delta, \ldots$ Formally we write this assumption as follows:

$(\mathrm{m})$ the transition probabilities $Q_{i j}^{(\delta)}(t), i, j \in \mathbb{X}$, as functions of $t$, may jump only at the moments $\delta, 2 \delta, \ldots$.

Choose $\sigma>\max _{i \in \mathbb{X}} \sigma_{i}$. In this case, $p_{i}^{(\delta)}$ and $q_{i}^{(\delta)}$ defined below in condition $\mathbf{G}_{\mathbf{1}}$ are positive numbers and their sum is less than 1 for all $i \in \mathbb{X}$ and

$$
0<\delta<\delta_{0}=\min _{i \in \mathbb{X}} \frac{\left(\sigma^{2}-\sigma_{i}^{2}\right) \wedge \sigma_{i}^{2}}{\mu_{i}^{2}+1}
$$

$\mathbf{G}_{\mathbf{1}}$ : The numbers $u_{i}^{(\delta)}, p_{i}^{(\delta)}$, and $q_{i}^{(\delta)}$ are such that

$$
\begin{gathered}
u_{i}^{(\delta)}=\sigma \sqrt{\delta}, \quad p_{i}^{(\delta)}=\frac{\sigma_{i}^{2}}{2 \sigma^{2}}+\frac{\mu_{i}}{2 \sigma} \sqrt{\delta}+\frac{\mu_{i}^{2}}{2 \sigma^{2}} \delta \\
q_{i}^{(\delta)}=\frac{\sigma_{i}^{2}}{2 \sigma^{2}}-\frac{\mu_{i}}{2 \sigma} \sqrt{\delta}+\frac{\mu_{i}^{2}}{2 \sigma^{2}} \delta
\end{gathered}
$$

for all $i \in \mathbb{X}$.

Condition $\mathbf{G}_{\mathbf{1}}$ implies that $\mathrm{E} Y_{0, i, 1}^{(\delta)}=\mu_{i} \delta$ and $\operatorname{Var} Y_{0, i, 1}^{(\delta)}=\sigma_{i}^{2} \delta$. It also implies the $J$-convergence of the processes $Y_{0, i}^{(\delta)}(t), t \in[0, T]$, to the processes $Y_{0, i}^{(0)}(t), t \in[0, T]$, as $\delta \rightarrow 0$, for all $i \in \mathbb{X}$. Moreover conditions $\mathbf{D}_{\mathbf{1}}$ and $\mathbf{D}_{\mathbf{2}}$ follow from $\mathbf{G}_{\mathbf{1}}$ for all $i \in \mathbb{X}$.

The moment generation function $\operatorname{E} \exp \left\{\beta Y_{0, i}^{(\delta)}(t)\right\}$ exists for all $\beta \in \mathbb{R}_{1}$ and can be written explicitly, namely,

$$
\mathrm{E} \exp \left\{\beta Y_{0, i}^{(\delta)}(t)\right\}= \begin{cases}\left(e^{\beta \sigma \sqrt{\delta}} p_{i}^{(\delta)}+r_{i}^{(\delta)}+e^{-\beta \sigma \sqrt{\delta}} q_{i}^{(\delta)}\right)^{[t / \delta]}, & \text { if } \delta>0 \\ e^{\beta \mu_{i} t+\beta^{2} \sigma_{i}^{2} t / 2}, & \text { if } \delta=0 .\end{cases}
$$

Thus condition $\mathbf{D}_{\mathbf{3}}$ holds for these processes for all $\beta^{\prime}>\beta$ and for all $i \in \mathbb{X}$, whence condition $\mathbf{F}_{\mathbf{2}}$ follows.

Assume that the semi-Markov stochastic index is a Markov chain whose transition probabilities are such that $Q_{i j}^{(\delta)}(t)=p_{i j}^{(\delta)} Q_{i}^{(\delta)}(t)$, where $Q_{i}^{(\delta)}(t)$ are geometric distributions of random sojourn times. This means that the sojourn times take values $n \delta$ with the probabilities

$$
Q_{i}^{(\delta)}\left(1-Q_{i}^{(\delta)}\right)^{n-1}, \quad n=1,2, \ldots
$$

In this case, condition $\mathbf{F}_{\mathbf{1}}$ follows from

$\mathbf{F}_{\mathbf{3}}:$ (a) $p_{i j}^{(\delta)} \rightarrow p_{i j}^{(0)}$ as $\delta \rightarrow 0, i, j \in \mathbb{X}$

(b) $Q_{i}^{(\delta)} / \delta \rightarrow \lambda_{i}>0$ as $\delta \rightarrow 0, i \in \mathbb{X}$. 
The limit transition probabilities become of the form:

$$
Q_{i j}^{(0)}(t)=p_{i j}^{(0)}\left(1-e^{-\lambda_{i} t}\right), \quad t \geq 0, i, j \in \mathbb{X} .
$$

Collecting together the above remarks, we conclude that Theorem 3 follows from conditions $\mathbf{A}_{\mathbf{1}}, \mathbf{A}_{\mathbf{4}}, \mathbf{F}_{\mathbf{1}}$ and $\mathbf{G}_{\mathbf{1}}$, that is,

$$
\Phi\left(\mathcal{M}_{\max , T}^{(\delta)}\right) \rightarrow \Phi\left(\mathcal{M}_{\max , T}^{(0)}\right) \quad \text { as } \delta \rightarrow 0
$$

for the exponential trinomial price process $S^{(\delta)}(t)=e^{Y^{(\delta)}(t)}$ introduced by equalities (42) and (46)- 477).

For the sake of simplicity assume that $\delta=T / N$ and consider the partition

$$
\Pi_{\delta}=\left\langle t_{0}=0<t_{1}=\delta<\cdots<t_{N-1}=(N-1) \delta<t_{N}=T\right\rangle
$$

of the interval $[0, T]$.

In this case, the Markov chain $\left(n, Y^{(\delta)}(n \delta), X^{(\delta)}(n \delta)\right), n=0,1, \ldots$, represents a trinomial tree model with the initial node $\left(0, y_{0}, i_{0}\right)$ and with $(2 n+1) m$ nodes of the form $\left(n, y_{\delta} \pm k \sigma \sqrt{\delta}, i\right), k=0,1, \ldots, n, i=1, \ldots, m$, after $n \geq 1$ steps.

The standard backward procedure can be applied to find the optimal expected reward at the moment 0 for the discrete time exponential trinomial price process with Markov modulation $\left(S^{(\delta)}(n \delta), X^{(\delta)}(n \delta)\right)$. This optimal expected reward coincides with the reward functional $\Phi\left(\mathcal{M}_{\Pi_{\delta}, T}^{(\delta)}\right)$ for the exponential trinomial price processes $S^{(\delta)}(t)=e^{Y^{(\delta)}(t)}$ introduced above in (42) and (46)-(47).

To estimate the difference $\Phi\left(\mathcal{M}_{\max , T}^{(\delta)}\right)-\Phi\left(\mathcal{M}_{\Pi_{\delta}, T}^{(\delta)}\right)$, we apply Theorem 1 . In this case, $d\left(\Pi_{\delta}\right)=\delta$ and

$$
\Delta_{\beta}\left(Y^{(\delta)}(\cdot), \delta, T\right)=\max _{i \in \mathbb{X}}\left(\operatorname{E} \exp \left\{\beta\left|Y_{1, i, 1}^{(\delta)}\right|\right\}-1\right) \leq e^{\beta \sigma \sqrt{\delta}}-1 .
$$

Theorem 1 yields the following estimate:

$$
\Phi\left(\mathcal{M}_{\max , T}^{(\delta)}\right)-\Phi\left(\mathcal{M}_{\Pi_{\delta}, T}^{(\delta)}\right) \leq L_{3} \delta+L_{4}\left(e^{\beta \sigma \sqrt{\delta}}-1\right)^{\frac{\beta-\gamma}{\beta}} \rightarrow 0 \quad \text { as } \delta \rightarrow 0 .
$$

Thus, Theorem 3 and conditions $\mathbf{A}_{\mathbf{1}}, \mathbf{A}_{\mathbf{4}}, \mathbf{F}_{\mathbf{3}}$, and $\mathbf{G}_{\mathbf{1}}$ imply that the optimal expected reward $\Phi\left(\mathcal{M}_{\Pi_{\delta}, T}^{(\delta)}\right)$ converges to the reward functional $\Phi\left(\mathcal{M}_{\max , T}^{(0)}\right)$ for the geometric Brownian motion defined in (42) and (47). This Brownian motion is modulated by the continuous time homogeneous Markov chain with transition probabilities given in (49).

We use the trinomial model instead of the standard binomial one to fit the corresponding values of the expectations and variances for price jumps $Y_{0, i, 1}^{(\delta)}$ by choosing

(n) some values of probabilities $p_{i}^{(\delta)}$ and $q_{i}^{(\delta)}, i \in \mathbb{X}$, and, at the same time,

(o) the same values of jumps $u_{i}^{(\delta)}=\sigma \sqrt{\delta}$ for all $i \in \mathbb{X}$.

The latter property (o) provides automatically the necessary recombining property for the corresponding trinomial tree model. The recombining property means that, for any node, two sequential up-down jumps (first up and then down) result in the same node of the tree as two sequential down-up jumps. This allows us to construct the tree model for the modulated log-price processes for which the number of nodes, as a function of the number of steps, has a linear rate of growth.

In the case of the approximation of a continuous type option with maturity $T$ by the corresponding discrete time model with the time step $\delta=T / N$, the corresponding tree has $N$ steps, and therefore $(2 N+1) m$ nodes after the last $N$ th step, $(2(N-1)+1) m$ nodes after the $(N-1)$ th step, etc. 
The above backward algorithm can also be generalized to the general case of a trinomial tree model with semi-Markov index $X^{(\delta)}(t)$ jumping at the moments $\delta, 2 \delta, \ldots$ only. In this case, the Markov chain

$$
\left(n, Y^{(\delta)}(n \delta), X^{(\delta)}(n \delta), T^{(\delta)}(n \delta)\right), \quad n=0,1, \ldots,
$$

is considered as a tree model with the initial node $\left(0, y_{0}, i_{0}, 0\right)$ and with $(2 n+1) m n$ nodes of the form $\left(n, y_{0} \pm k \sigma \sqrt{\delta}, i, l\right), k=0,1, \ldots, n, i=1, \ldots, m, l=0,1, \ldots, n$, after $n \geq 1$ steps.

In the case under consideration, the number of nodes of the corresponding tree, as a function of the number of steps, has a quadratic rate of growth. In the case of the approximation of the continuous type option with maturity $T$ by the corresponding discrete time model with the time step $\delta=1 / N$, the corresponding tree has $N$ steps, and therefore $(2 N+1) m N$ nodes after the last $N$ th step, $(2(N-1)+1) m(N-1)$ nodes after the $(N-1)$ th step, etc.

Theorem 3 and conditions $\mathbf{A}_{\mathbf{1}}, \mathbf{A}_{\mathbf{4}}, \mathbf{F}_{\mathbf{1}}$, and $\mathbf{G}_{\mathbf{1}}$ guaranty that these optimal expected rewards at time 0 converge to the corresponding optimal expected reward functional $\Phi\left(\mathcal{M}_{\max , T}^{(0)}\right)$ of the geometric Brownian motion in the case of the trinomial tree model with semi-Markov modulation described above. The Brownian motion defined in (42) and (47) is modulated by a semi-Markov index whose transition probabilities

$$
Q_{i j}^{(0)}(t)=p_{i j}^{(0)} F_{i j}^{(0)}(t)
$$

are given in condition $\mathbf{F}_{\mathbf{1}}$.

Numerical algorithms for the approximation of the optimal reward values for exponential price processes modulated by stochastic market indices require a more detailed consideration. The corresponding results will be presented in a separate publication.

In conclusion, we would like to mention that similar examples for multivariate exponential price processes with independent increments are considered in Lundgren, Silvestrov, and Kukush [7].

\section{BIBLIOGRAPHY}

1. D. S. Silvestrov, H. Jönsson, and F. Stenberg, Convergence of option rewards for Markov type price processes modulated by stochastic indices. I, Teor. İmovir. ta Matem. Statyst. 79 (2009), 138-154. MR2494545 (2010e:60151)

2. Y. S. Chow, H. Robbins, and D. Siegmund, The Theory of Optimal Stopping, Houghton Mifflin Co., Boston, 1971; Dover, New York, 1991. MR 1105325 (92b:62115)

3. H. Jönsson, Monte Carlo studies of American type options with discrete time, Theory Stoch. Process. 7(23) (2001), no. 1-2, 163-188.

4. H. Jönsson, Optimal Stopping Domains and Reward Functions for Discrete Time American Type Options, Ph.D. Thesis, vol. 22, Mälardalen University, 2005.

5. H. Jönsson, A. G. Kukush, and D. S. Silvestrov, Threshold structure of optimal stopping strategies for American type options. I, Theor. Ǐmovir. ta Matem. Statyst. 71 (2004), 113-123; English transl. in Theory Probab. Math. Statist. 71 (2005), 93-103. MR2144323 (2006h:91075)

6. H. Jönsson, A. G. Kukush, and D. S. Silvestrov, Threshold structure of optimal stopping strategies for American type options. II, Theor. Imovir. ta Matem. Statyst. 72 (2005), 42-53; English transl. in Theory Probab. Math. Statist. 72 (2006), 47-58. MR2168135 (2006i:62070)

7. R. Lundgren, D. S. Silvestrov, and A. G. Kukush, Reselling of options and convergence of option rewards, J. Numer. Appl. Math. 1(96) (2008), 149-172.

8. A. G. Kukush and D. S. Silvestrov, Skeleton approximation of optimal stopping strategies for American type options with continuous time, Theory Stoch. Process. 7(23) (2001), no. 1-2, $215-230$.

9. A. G. Kukush and D. S. Silvestrov, Optimal price of American type options with discrete time, Theory Stoch. Process. 10(26) (2004), no. 1-2, 72-96. MR2327852 
10. A. N. Shiryaev, Sequential Analysis. Optimal Stopping Rules, Nauka, Moscow, 1976; English edition, Optimal Stopping Rules, Springer, New York, 1978. MR0445744(56:4078), MR0468067 (57:7906)

11. D. S. Silvestrov, Limit Theorems for Randomly Stopped Stochastic Processes, Springer, London, 2004. MR2030998 (2005b:60003)

12. D. Silvestrov, V. Galochkin, and A. Malyarenko, OPTAN - a pilot program system for analysis of options, Theory Stoch. Process. 7(23) (2001), no. 1-2, 291-300.

13. D. S. Silvestrov, V. G. Galochkin, and V. G. Sibirtsev, Algorithms and programs for optimal Monte Carlo pricing of American type options, Theory Stoch. Process. 5(21) (1999), no. 1-2, $175-187$.

14. A. V. Skorokhod, Limit theorems for stochastic processes, Teor. Veroyatn. Primen. 1 (1956), 289-319; English transl. in Theory Probab. Appl. 1, 261-290. MR0084897 (18:943c)

15. A. V. Skorokhod, Random Processes with Independent Increments, Nauka, Moscow, 1964; English edition, Kluwer, Dordrecht, 1991. MR860563 (88b:60171) MR1155400 (93a:60114)

16. F. Stenberg, Semi-Markov Models for Insurance and Option Rewards, Ph.D. Thesis, vol. 38, Mälardalen University, 2006.

Mälardalen University, VÄsterås, Sweden

E-mail address: dmitrii.silvestrov@mdh.se

Eurandom, Eindhoven University of Technology, The Netherlands

E-mail address: jonsson.@eurandom.tue.nl

Nordea Bank, Stockholm, Sweden

E-mail address: fredsten@kth.se

Received 25/AUG/2008

English original provided by the authors 